Bull. Korean Math. Soc. 50 (2013), No. 3, pp. 761-776

http://dx.doi.org/10.4134/BKMS.2013.50.3.761

\title{
ON SOME MODULAR EQUATIONS AND THEIR APPLICATIONS I
}

Jinhee Yi, Man Gi Cho, Jeong Hwan Kim, Seong Hoi Lee, Jae Myung Yu, And Dae Hyun Paek

\begin{abstract}
We derive several modular equations and present their proofs based on concise algebraic computations. In addition, we establish explicit relations and formulas for some parameterizations for the theta functions $\varphi$ and $\psi$ and show some applications of the modular equations to evaluations of the cubic continued fraction and the theta function $\psi$.
\end{abstract}

\section{Introduction}

We begin this section by introducing Ramanujan's definition of his general theta function. For $|a b|<1$, define

$$
f(a, b):=\sum_{n=-\infty}^{\infty} a^{n(n+1) / 2} b^{n(n-1) / 2} .
$$

Note that two special cases of $f(a, b)$ are defined by, for $|q|<1$,

$$
\varphi(q):=f(q, q)=\sum_{n=-\infty}^{\infty} q^{n^{2}}=\left(-q ; q^{2}\right)_{\infty}^{2}\left(q^{2} ; q^{2}\right)_{\infty}
$$

and

$$
\psi(q):=f\left(q, q^{3}\right)=\sum_{n=0}^{\infty} q^{n(n+1) / 2}=\frac{\left(q^{2} ; q^{2}\right)_{\infty}}{\left(q ; q^{2}\right)_{\infty}}
$$

where

$$
(a ; q)_{\infty}:=\prod_{n=0}^{\infty}\left(1-a q^{n}\right) .
$$

Let $a, b$, and $c$ be arbitrary complex numbers except that $c$ cannot be a nonpositive integer. Then, for $|z|<1$, the Gaussian or ordinary hypergeometric

Received February 16, 2012.

2010 Mathematics Subject Classification. Primary 11F27, 33C90; Secondary 11F20, 33C05, 33C75.

Key words and phrases. theta functions, modular equations. 
function ${ }_{2} F_{1}(a, b ; c ; z)$ is defined by

$$
{ }_{2} F_{1}(a, b ; c ; z):=\sum_{n=0}^{\infty} \frac{(a)_{n}(b)_{n}}{(c)_{n} n !} z^{n},
$$

where $(a)_{0}:=1$ and $(a)_{n}:=a(a+1)(a+2) \cdots(a+n-1)$ for each positive integer $n$.

Now the complete elliptic integral of the first kind $K(k)$ is defined by

$$
K(k):=\int_{0}^{\pi / 2} \frac{d \theta}{\sqrt{1-k^{2} \sin ^{2} \theta}}=\frac{\pi}{2}{ }_{2} F_{1}\left(\frac{1}{2}, \frac{1}{2} ; 1 ; k^{2}\right)=\frac{\pi}{2} \varphi^{2}\left(e^{-\pi \frac{K^{\prime}}{K}}\right),
$$

where $0<k<1, K^{\prime}=K\left(k^{\prime}\right)$, and $k^{\prime}=\sqrt{1-k^{2}}$. The number $k$ is called the modulus of $K$ and $k^{\prime}$ is called the complementary modulus.

Let $K, K^{\prime}, L$, and $L^{\prime}$ denote complete elliptic integrals of the first kind associated with the moduli $k, k^{\prime}, l$, and $l^{\prime}$, respectively, where $0<k<1$ and $0<l<1$. Suppose that

$$
\frac{L^{\prime}}{L}=n \frac{K^{\prime}}{K}
$$

holds for some positive integer $n$. A relation between $k$ and $l$ induced by (1.2) is called a modular equation of degree $n$.

If we set

$$
q=\exp \left(-\pi \frac{K^{\prime}}{K}\right) \quad \text { and } \quad q^{\prime}=\exp \left(-\pi \frac{L^{\prime}}{L}\right),
$$

we see that (1.2) is equivalent to the relation $q^{n}=q^{\prime}$. Thus, a modular equation can be viewed as an identity involving theta functions at the arguments $q$ and $q^{n}$.

Note that the definition of a modular equation mentioned above is the one used by Ramanujan, but we emphasize that there are several definitions of a modular equation in the literature. For example, refer the books by R. A. Rankin in [5] and B. Schoeneberg in [6] for other definitions of a modular equation. Following Ramanujan, set $\alpha=k^{2}$ and $\beta=l^{2}$, then we say that $\beta$ has degree $n$ over $\alpha$. By the relationship between complete elliptic integrals of the first kind and hypergeometric function, we have

$$
n \frac{{ }_{2} F_{1}\left(\frac{1}{2}, \frac{1}{2} ; 1 ; 1-\alpha\right)}{{ }_{2} F_{1}\left(\frac{1}{2}, \frac{1}{2} ; 1 ; \alpha\right)}=\frac{{ }_{2} F_{1}\left(\frac{1}{2}, \frac{1}{2} ; 1 ; 1-\beta\right)}{{ }_{2} F_{1}\left(\frac{1}{2}, \frac{1}{2} ; 1 ; \beta\right)} .
$$

Let $z_{n}=\varphi^{2}\left(q^{n}\right)$. Then the multiplier $m$ for degree $n$ is defined by

$$
m:=\frac{\varphi^{2}(q)}{\varphi^{2}\left(q^{n}\right)}=\frac{z_{1}}{z_{n}} .
$$

Next we introduce 4 parameterizations for the theta functions $\varphi$ and $\psi$ from $[7,8,9]$. For any positive real numbers $k$ and $n$, define $h_{k, n}$ by

$$
h_{k, n}:=\frac{\varphi(q)}{k^{1 / 4} \varphi\left(q^{k}\right)},
$$


where $q=e^{-\pi \sqrt{n / k}}$, define $h_{k, n}^{\prime}$ by

$$
h_{k, n}^{\prime}:=\frac{\varphi(-q)}{k^{1 / 4} \varphi\left(-q^{k}\right)},
$$

where $q=e^{-2 \pi \sqrt{n / k}}$, define $l_{k, n}$ by

$$
l_{k, n}:=\frac{\psi(-q)}{k^{1 / 4} q^{(k-1) / 8} \psi\left(-q^{k}\right)},
$$

where $q=e^{-\pi \sqrt{n / k}}$, define $l_{k, n}^{\prime}$ by

$$
l_{k, n}^{\prime}:=\frac{\psi(q)}{k^{1 / 4} q^{(k-1) / 8} \psi\left(q^{k}\right)},
$$

where $q=e^{-\pi \sqrt{n / k}}$.

Moreover, in $[7,8,9]$, several new modular equations for the theta functions were derived, some explicit relations and formulas for the parameterizations were offered, and some values of the parameterizations were determined. In this paper, we further derive some modular equations for the theta functions $\varphi$ and $\psi$ and present their proofs based on concise algebraic computations, find explicit relations and formulas for the corresponding parameterizations, and evaluate values of $h_{k, n}, h_{k, n}^{\prime}, l_{k, n}$, and $l_{k, n}^{\prime}$ for some positive real numbers $k$ and $n$ by employing the relations and formulas established earlier.

We are now in position to describe the scope of this study. In Section 2 , we introduce basic theta function identities that will be useful in deriving modular equations. In Section 3, we derive some modular equations and find corresponding explicit relations or formulas for $h_{k, n}, h_{k, n}^{\prime}, l_{k, n}$, and $l_{k, n}^{\prime}$ by employing these modular equations. In Section 4 , we evaluate specific values of $h_{k, n}, h_{k, n}^{\prime}, l_{k, n}$, and $l_{k, n}^{\prime}$ for some positive real numbers $k$ and $n$ based on the explicit relations and formulas established in Section 3. Finally, in Section 5, we consider some applications of the modular equations to evaluations of the cubic continued fraction and the theta function $\psi$.

\section{Preliminary results}

In this section, we introduce basic theta function identities that will play key roles in deriving some modular equations. Let $k$ be the modulus as in (1.1). Set $x=k^{2}$ and also set

$$
k^{2}=x=1-\frac{\varphi^{4}(-q)}{\varphi^{4}(q)} .
$$

Then

$$
\varphi^{2}(q)={ }_{2} F_{1}\left(\frac{1}{2}, \frac{1}{2} ; 1 ; x\right)=: z
$$


where

$$
q=e^{-y}:=\exp \left(-\pi \frac{{ }_{2} F_{1}\left(\frac{1}{2}, \frac{1}{2} ; 1 ; 1-x\right)}{{ }_{2} F_{1}\left(\frac{1}{2}, \frac{1}{2} ; 1 ; x\right)}\right)=\exp \left(-\pi \frac{K\left(k^{\prime}\right)}{K(k)}\right) .
$$

Lemma 2.1 ([1], Theorem 5.4.1). If $x, q$, and $z$ are related by (2.1), (2.2), and (2.3), then

(i) $\varphi(q)=\sqrt{z}$

(ii) $\varphi(-q)=\sqrt{z}(1-x)^{1 / 4}$,

(iii) $\varphi\left(-q^{2}\right)=\sqrt{z}(1-x)^{1 / 8}$.

Lemma 2.2 ([1], Theorem 5.4.2). If $x, q$, and $z$ are related by (2.1), (2.2), and (2.3), then
(i) $\psi(q)=\sqrt{\frac{1}{2} z}\left(\frac{x}{q}\right)^{1 / 8}$,
(ii) $\psi(-q)=\sqrt{\frac{1}{2} z}\left(\frac{x(1-x)}{q}\right)^{1 / 8}$,
(iii) $\psi\left(q^{2}\right)=\frac{1}{2} \sqrt{z}\left(\frac{x}{q}\right)^{1 / 4}$.

Lemma 2.3 ([2], Entry 1(iii), Chapter 20). For $|q|<1$, we have

$$
\frac{\varphi\left(q^{1 / 3}\right)}{\varphi\left(q^{3}\right)}=1+\left(\frac{\varphi^{4}(q)}{\varphi^{4}\left(q^{3}\right)}-1\right)^{1 / 3} .
$$

Lemma 2.4 ([2], Entry 3, Chapter 20). Let $\beta$ and $\gamma$ be the third and ninth degrees, respectively. Let $m=\frac{z_{1}}{z_{3}}$ and $m^{\prime}=\frac{z_{3}}{z_{9}}$. Then

(i) $1+4^{1 / 3}\left(\frac{\alpha^{3}(1-\alpha)^{3}}{\beta(1-\beta)}\right)^{1 / 24}=\frac{3}{\sqrt{m m^{\prime}}}$,

(ii) $1+4^{1 / 3}\left(\frac{\gamma^{3}(1-\gamma)^{3}}{\beta(1-\beta)}\right)^{1 / 24}=\sqrt{m m^{\prime}}$,

(iii) $1-2^{4 / 3}\left(\frac{\alpha^{3} \gamma^{3}(1-\alpha)^{3}(1-\gamma)^{3}}{\beta^{2}(1-\beta)^{2}}\right)^{1 / 24}=\frac{m^{\prime}}{m}$,

(iv) $\left(\frac{\gamma}{\alpha}\right)^{1 / 8}+\left(\frac{1-\gamma}{1-\alpha}\right)^{1 / 8}-\left(\frac{\gamma(1-\gamma)}{\alpha(1-\alpha)}\right)^{1 / 8}=\sqrt{m m^{\prime}}$,

(v) $\left(\frac{\alpha}{\gamma}\right)^{1 / 8}+\left(\frac{1-\alpha}{1-\gamma}\right)^{1 / 8}-\left(\frac{\alpha(1-\alpha)}{\gamma(1-\gamma)}\right)^{1 / 8}=\frac{3}{\sqrt{m m^{\prime}}}$.

Lemma 2.5 ([2], Entry 5, Chapter 19). Let $\beta$ be the third degree and $m=\frac{z_{1}}{z_{3}}$. Then

(i) $\sqrt{\frac{\beta}{\alpha}}+\sqrt{\frac{1-\beta}{1-\alpha}}-\sqrt{\frac{\beta(1-\beta)}{\alpha(1-\alpha)}}=m^{2}$, 
(ii) $\sqrt{\frac{\alpha}{\beta}}+\sqrt{\frac{1-\alpha}{1-\beta}}-\sqrt{\frac{\alpha(1-\alpha)}{\beta(1-\beta)}}=\left(\frac{3}{m}\right)^{2}$.

Lemma 2.6 ([8], Theorem 2.2). For any positive real number $k$,

$$
h_{k, 1}=1 \text {. }
$$

Lemma 2.7 ([9], Theorem 2.3). For any positive real number $k$,

$$
l_{k, 1}=1 \text {. }
$$

The following results exhibit some general formulas for the Ramanujan's cubic continued fractions $G\left(e^{-\pi \sqrt{n}}\right)$ and $G\left(-e^{-\pi \sqrt{n}}\right)$ in terms of $h_{k, n}, h_{k, n}^{\prime}$, $l_{k, n}$, and $l_{k, n}^{\prime}$.

Lemma 2.8 ([9], Theorem 6.2). For any positive real number $n$, we have

(i) $G\left(e^{-\pi \sqrt{n}}\right)=\frac{1}{\sqrt{3} l_{9, n}^{\prime}-1}$,

(ii) $G^{3}\left(e^{-\pi \sqrt{n / 3}}\right)=\frac{1}{3 l_{3, n}^{4}-1}$,

(iii) $G\left(e^{-2 \pi \sqrt{n}}\right)=\frac{1-\sqrt{3} h_{9, n}^{\prime}}{2}$,

(iv) $G\left(-e^{-\pi \sqrt{n}}\right)=\frac{\frac{2}{1}}{\sqrt{3} l_{9, n}+1}$,

(v) $G\left(-e^{-\pi \sqrt{n}}\right)=\frac{1-\sqrt{3} h_{9, n}}{2}$.

Note that $G\left(-e^{-\pi \sqrt{n}}\right)$ can be evaluated by using either $l_{9, n}$ or $h_{9, n}$. Moreover, from Lemma $2.8(\mathrm{iv})$ and $(\mathrm{v})$, we find that $\left(\sqrt{3} h_{9, n}-1\right)\left(\sqrt{3} l_{9, n}+1\right)=2$. This is the same relation obtained in Corollary 3.10, which will be discussed later.

Lemma 2.9 ([9], Theorem 5.2). Let $a=\frac{\pi^{1 / 4}}{\Gamma(3 / 4)}$. Then, for every positive real number $n$, we have

(i) $\psi\left(e^{-n \pi}\right)=\frac{a e^{n \pi / 8}}{2^{5 / 8} n^{1 / 4} l_{n, n}^{\prime}}$,

(ii) $\psi\left(-e^{-n \pi}\right)=\frac{a e^{n \pi / 8}}{2^{3 / 4} n^{1 / 4} l_{n, n}}$.

\section{Modular equations}

In [3], there are Ramanujan's 23 eta function identities, which are certain types of modular equations. In this section, we derive some modular equations and present their proofs based on concise algebraic computations by employing the theory of theta functions in the spirit of Ramanujan. In addition, we establish corresponding explicit relations and formulas for $h_{k, n}, h_{k, n}^{\prime}, l_{k, n}$, and $l_{k, n}^{\prime}$ by employing these modular equations. 
Theorem 3.1. If $P=\frac{\varphi(q)}{\varphi\left(q^{9}\right)}$ and $Q=\frac{\varphi(-q)}{\varphi\left(-q^{9}\right)}$, then

$$
\sqrt{P Q}+\frac{3}{\sqrt{P Q}}=\sqrt{\frac{Q}{P}}+\sqrt{\frac{P}{Q}}+2 .
$$

Proof. By Lemma 2.1(i) and (ii),

$$
P=\sqrt{\frac{z_{1}}{z_{9}}} \text { and } Q=\sqrt{\frac{z_{1}}{z_{9}}}\left(\frac{1-\alpha}{1-\gamma}\right)^{1 / 4},
$$

where $\gamma$ has degree 9 over $\alpha$. Thus

$$
\frac{Q}{P}=\left(\frac{1-\alpha}{1-\gamma}\right)^{1 / 4}
$$

By Lemma 2.4(iv) and (v),

$$
\left(\frac{\gamma}{\alpha}\right)^{1 / 8}+\sqrt{\frac{P}{Q}}-\left(\frac{\gamma}{\alpha}\right)^{1 / 8} \sqrt{\frac{P}{Q}}=P
$$

and

$$
\left(\frac{\alpha}{\gamma}\right)^{1 / 8}+\sqrt{\frac{Q}{P}}-\left(\frac{\alpha}{\gamma}\right)^{1 / 8} \sqrt{\frac{Q}{P}}=\frac{3}{P}
$$

Combining above two equations in terms of $P$ and $Q$, we deduce that

$$
\frac{P-\sqrt{\frac{P}{Q}}}{1-\sqrt{\frac{P}{Q}}}=\frac{1-\sqrt{\frac{Q}{P}}}{\frac{3}{P}-\sqrt{\frac{Q}{P}}} .
$$

This then completes the proof.

Using the definitions of $h_{k, n}$ and $h_{k, n}^{\prime}$, we have the following:

Corollary 3.2. For every positive real number $n$, we have

$$
\sqrt{3}\left(\sqrt{h_{9, n} h_{9, n / 4}^{\prime}}+\frac{1}{\sqrt{h_{9, n} h_{9, n / 4}^{\prime}}}\right)=\sqrt{\frac{h_{9, n}}{h_{9, n / 4}^{\prime}}}+\sqrt{\frac{h_{9, n / 4}^{\prime}}{h_{9, n}}}+2 .
$$

Proof. Letting $q=e^{-\pi \sqrt{n / 9}}$ in (1.3) and (1.4), we find that $P=\sqrt{3} h_{9, n}$ and $Q=\sqrt{3} h_{9, n / 4}^{\prime}$ in Theorem 3.1. Rewriting (3.1) in terms of $h_{9, n}$ and $h_{9, n / 4}^{\prime}$, we complete the proof.

Theorem 3.3. If $P=\frac{\varphi(q)}{\varphi\left(q^{9}\right)}$ and $Q=\frac{\varphi\left(-q^{2}\right)}{\varphi\left(-q^{18}\right)}$, then

$$
\frac{P}{Q}+\frac{Q}{P}+2=Q+\frac{3}{Q} \text {. }
$$


Proof. By Lemma 2.1(i) and (iii),

where $\gamma$ has degree 9 over $\alpha$. Thus

$$
P=\sqrt{\frac{z_{1}}{z_{9}}} \text { and } Q=\sqrt{\frac{z_{1}}{z_{9}}}\left(\frac{1-\alpha}{1-\gamma}\right)^{1 / 8},
$$

$$
\frac{Q}{P}=\left(\frac{1-\alpha}{1-\gamma}\right)^{1 / 8}
$$

By Lemma 2.4(iv) and (v),

$$
\left(\frac{\gamma}{\alpha}\right)^{1 / 8}+\frac{P}{Q}-\left(\frac{\gamma}{\alpha}\right)^{1 / 8} \frac{P}{Q}=P
$$

and

$$
\left(\frac{\alpha}{\gamma}\right)^{1 / 8}+\frac{Q}{P}-\left(\frac{\alpha}{\gamma}\right)^{1 / 8} \frac{Q}{P}=\frac{3}{P} .
$$

Combining above two equations in terms of $P$ and $Q$ as in the proof of Theorem 3.1 , we complete the proof.

Using the definitions of $h_{k, n}$ and $h_{k, n}^{\prime}$, we have the following:

Corollary 3.4. For every positive real number $n$, we have

$$
\sqrt{3}\left(h_{9, n}^{\prime}+\frac{1}{h_{9, n}^{\prime}}\right)=\frac{h_{9, n}}{h_{9, n}^{\prime}}+\frac{h_{9, n}^{\prime}}{h_{9, n}}+2 .
$$

Proof. Letting $q=e^{-\pi \sqrt{n / 9}}$ in (1.3) and (1.4), we find that $P=\sqrt{3} h_{9, n}$ and $Q=\sqrt{3} h_{9, n}^{\prime}$ in Theorem 3.3. Rewriting (3.3) in terms of $h_{9, n}$ and $h_{9, n}^{\prime}$, we complete the proof.

Theorem 3.5. If $P=\frac{\varphi(q)}{\varphi\left(q^{9}\right)}$ and $Q=\frac{\varphi\left(q^{3}\right)}{\varphi\left(q^{27}\right)}$, then

$$
\left(P-3+\frac{3}{P}\right)\left(Q-3+\frac{3}{Q}\right)=\left(\frac{Q}{P}\right)^{2} .
$$

Proof. Let $A=\varphi\left(q^{9}\right)$ and $B=\varphi\left(q^{27}\right)$. Then $\varphi(q)=A P$ and $\varphi\left(q^{3}\right)=B Q$. Thus by Lemma 2.3,

$$
P-1=\left(\frac{B^{4} Q^{4}}{A^{4}}-1\right)^{1 / 3} \text { and } Q-1=\left(\frac{A^{4}}{B^{4}}-1\right)^{1 / 3} .
$$

Combining and rewriting above two equations in terms of $P$ and $Q$, we deduce that

$$
\left((P-1)^{3}+1\right)\left((Q-1)^{3}+1\right)=Q^{4} .
$$

Dividing both sides of this equation by $P^{2} Q^{2}$, we complete the proof.

Using the definition of $h_{k, n}$, we have the following: 
Corollary 3.6. For every positive real number $n$, we have

$$
3 h_{9, n}\left(h_{9, n}^{2}-\sqrt{3} h_{9, n}+1\right)\left(h_{9,9 n}^{2}-\sqrt{3} h_{9,9 n}+1\right)=h_{9,9 n}^{3} .
$$

Proof. Letting $q=e^{-\pi \sqrt{n / 9}}$ in (1.3), we find that $P=\sqrt{3} h_{9, n}$ and $Q=$ $\sqrt{3} h_{9,9 n}$ in Theorem 3.5. Rewriting (3.5) in terms of $h_{9, n}$ and $h_{9,9 n}$, we complete the proof.

Theorem 3.7. If $P=\frac{\varphi(q)}{\varphi\left(q^{9}\right)}$ and $Q=\frac{\psi(q)}{q \psi\left(q^{9}\right)}$, then

$$
\frac{P}{Q}+\frac{Q}{P}+2=Q+\frac{3}{Q} \text {. }
$$

Proof. By Lemmas 2.1(i) and 2.2(i),

$$
P=\sqrt{\frac{z_{1}}{z_{9}}} \text { and } Q=\sqrt{\frac{z_{1}}{z_{9}}}\left(\frac{\alpha}{\gamma}\right)^{1 / 8},
$$

where $\gamma$ has degree 9 over $\alpha$. Thus

$$
\frac{Q}{P}=\left(\frac{\alpha}{\gamma}\right)^{1 / 8} \text {. }
$$

By Lemma 2.4(iv) and (v),

$$
\frac{P}{Q}+\left(\frac{1-\gamma}{1-\alpha}\right)^{1 / 8}-\frac{P}{Q}\left(\frac{1-\gamma}{1-\alpha}\right)^{1 / 8}=P
$$

and

$$
\frac{Q}{P}+\left(\frac{1-\alpha}{1-\gamma}\right)^{1 / 8}-\frac{Q}{P}\left(\frac{1-\alpha}{1-\gamma}\right)^{1 / 8}=\frac{3}{P}
$$

Combining above two equations in terms of $P$ and $Q$ as in the proof of Theorem 3.1 , we complete the proof.

Using the definitions of $h_{k, n}$ and $l_{k, n}^{\prime}$, we have the following:

Corollary 3.8. For every positive real number $n$, we have

$$
\sqrt{3}\left(l_{9, n}^{\prime}+\frac{1}{l_{9, n}^{\prime}}\right)=\frac{h_{9, n}}{l_{9, n}^{\prime}}+\frac{l_{9, n}^{\prime}}{h_{9, n}}+2 .
$$

Proof. Letting $q=e^{-\pi \sqrt{n / 9}}$ in (1.3) and (1.6), we find that $P=\sqrt{3} h_{9, n}$ and $Q=\sqrt{3} l_{9, n}^{\prime}$ in Theorem 3.7. Rewriting (3.7) in terms of $h_{9, n}$ and $l_{9, n}^{\prime}$, we complete the proof.

Theorem 3.9. If $P=\frac{\varphi(q)}{\varphi\left(q^{9}\right)}$ and $Q=\frac{\psi(-q)}{q \psi\left(-q^{9}\right)}$, then

$$
P(Q+1)=Q+3 \text {. }
$$


Proof. By Lemmas 2.1(i) and 2.2(ii),

$$
P=\sqrt{\frac{z_{1}}{z_{9}}} \text { and } Q=\sqrt{\frac{z_{1}}{z_{9}}}\left(\frac{\alpha(1-\alpha)}{\gamma(1-\gamma)}\right)^{1 / 8},
$$

where $\gamma$ has degree 9 over $\alpha$. Thus

$$
\frac{Q}{P}=\left(\frac{\alpha(1-\alpha)}{\gamma(1-\gamma)}\right)^{1 / 8}
$$

From the proofs of Lemma 2.4(i), (ii), and (iii), we know that $\frac{z_{1}}{z_{9}}, \alpha(1-\alpha)$, and $\gamma(1-\gamma)$ are expressible as rational functions of a parameter $t$ :

$$
\begin{gathered}
\frac{z_{1}}{z_{9}}=(1+2 t)^{2}, \\
\alpha(1-\alpha)=16 t\left(\frac{1-t}{1+2 t}\right)^{8} \frac{1-t^{3}}{1+8 t^{3}},
\end{gathered}
$$

and

$$
\gamma(1-\gamma)=16 t^{9}\left(\frac{1-t^{3}}{1+8 t^{3}}\right)
$$

Hence we find that

$$
P=1+2 t \quad \text { and } \quad \frac{Q}{P}=\frac{1-t}{t(1+2 t)} .
$$

Combining and rewriting above two equations in terms of $P$ and $Q$, we deduce that

This then completes the proof.

$$
\frac{Q}{P}=\frac{3-P}{P^{2}-P}
$$

Using the definitions of $h_{k, n}$ and $l_{k, n}$, we have the following:

Corollary 3.10. For every positive real number $n$, we have

$$
\left(\sqrt{3} h_{9, n}-1\right)\left(\sqrt{3} l_{9, n}+1\right)=2 .
$$

Proof. Letting $q=e^{-\pi \sqrt{n / 9}}$ in (1.3) and (1.5), we find that $P=\sqrt{3} h_{9, n}$ and $Q=\sqrt{3} l_{9, n}$ in Theorem 3.9. Rewriting (3.9) in terms of $h_{9, n}$ and $l_{9, n}$, we complete the proof.

Theorem 3.11. If $P=\frac{\psi(q)}{q^{1 / 4} \psi\left(q^{3}\right)}$ and $Q=\frac{\psi(-q)}{q^{1 / 4} \psi\left(-q^{3}\right)}$, then

$$
\left(P^{4}-Q^{4}-9\right)\left(\frac{1}{P^{4}}-\frac{1}{Q^{4}}-1\right)=1 \text {. }
$$

Proof. By Lemma 2.2(i) and (ii),

$$
P=\sqrt{\frac{z_{1}}{z_{3}}}\left(\frac{\alpha}{\beta}\right)^{1 / 8} \text { and } Q=\sqrt{\frac{z_{1}}{z_{3}}}\left(\frac{\alpha(1-\alpha)}{\beta(1-\beta)}\right)^{1 / 8}
$$


where $\beta$ has degree 3 over $\alpha$. Let

$$
m=\frac{z_{1}}{z_{3}} \text { and } \quad T=\sqrt{\frac{z_{1}}{z_{3}}}\left(\frac{1-\alpha}{1-\beta}\right)^{1 / 4} .
$$

Then

$$
\sqrt{\frac{\beta}{\alpha}}=\left(\frac{\sqrt{m}}{P}\right)^{4}, \quad \sqrt{\frac{1-\beta}{1-\alpha}}=\left(\frac{\sqrt{m}}{T}\right)^{2}, \quad \text { and } \quad \sqrt{\frac{\beta(1-\beta)}{\alpha(1-\alpha)}}=\left(\frac{\sqrt{m}}{Q}\right)^{4} .
$$

By Lemma 2.5(i) and (ii), we deduce that

$$
m=\frac{m}{P^{4}}+\frac{1}{T^{2}}-\frac{m}{Q^{2}}
$$

and

$$
\frac{9}{m}=\frac{P^{4}}{m}+T^{2}-\frac{Q^{4}}{m} .
$$

Combining above two equations in terms of $P$ and $Q$, we complete the proof.

Using the definitions of $l_{k, n}$ and $l_{k, n}^{\prime}$, we have the following:

Corollary 3.12. For every positive real number $n$, we have

$$
\left(l_{3, n}^{4}-l_{3, n}^{\prime 4}+3\right)\left(\frac{1}{l_{3, n}^{4}}-\frac{1}{l_{3, n}^{\prime 4}}+3\right)=1 .
$$

Proof. Letting $q=e^{-\pi \sqrt{n / 3}}$ in (1.5) and (1.6), we find that $P=3^{1 / 4} l_{3, n}^{\prime}$ and $Q=3^{1 / 4} l_{3, n}$ in Theorem 3.11. Rewriting (3.11) in terms of $l_{3, n}$ and $l_{3, n}^{\prime}$, we complete the proof.

Theorem 3.13. If $P=\frac{\psi(q)}{q \psi\left(q^{9}\right)}$ and $Q=\frac{\psi(-q)}{q \psi\left(-q^{9}\right)}$, then

$$
(P-Q-3)\left(\frac{1}{P}-\frac{1}{Q}-1\right)=1 .
$$

Proof. By Lemma 2.2(i) and (ii),

$$
P=\sqrt{\frac{z_{1}}{z_{9}}}\left(\frac{\alpha}{\gamma}\right)^{1 / 8} \text { and } Q=\sqrt{\frac{z_{1}}{z_{9}}}\left(\frac{\alpha(1-\alpha)}{\gamma(1-\gamma)}\right)^{1 / 8},
$$

where $\gamma$ has degree 9 over $\alpha$. Let $m=\frac{z_{1}}{z_{9}}$. Then we find that

$$
P=\sqrt{m}\left(\frac{\alpha}{\gamma}\right)^{1 / 8} \text { and } \frac{Q}{P}=\left(\frac{1-\alpha}{1-\gamma}\right)^{1 / 8} .
$$

By Lemma 2.4(iv) and (v), we deduce that

$$
\left(\frac{\gamma}{\alpha}\right)^{1 / 8}+\frac{P}{Q}-\left(\frac{\gamma}{\alpha}\right)^{1 / 8} \frac{P}{Q}=\sqrt{m}
$$


and

$$
\left(\frac{\alpha}{\gamma}\right)^{1 / 8}+\frac{Q}{P}-\left(\frac{\alpha}{\gamma}\right)^{1 / 8} \frac{Q}{P}=\frac{3}{\sqrt{m}}
$$

Since $\left(\frac{\alpha}{\gamma}\right)^{1 / 8}=\frac{P}{\sqrt{m}}$, above two equations can be rewritten as

$$
\frac{\sqrt{m}}{P}+\frac{P}{Q}-\frac{\sqrt{m}}{Q}=\sqrt{m} \text { and } \frac{P}{\sqrt{m}}+\frac{Q}{P}-\frac{Q}{\sqrt{m}}=\frac{3}{\sqrt{m}} .
$$

Combining and rewriting these two equations in terms of $P$ and $Q$, we complete the proof.

Using the definitions of $l_{k, n}$ and $l_{k, n}^{\prime}$, we have the following:

Corollary 3.14. For every positive real number $n$, we have

$$
\left(l_{9, n}-l_{9, n}^{\prime}+\sqrt{3}\right)\left(\frac{1}{l_{9, n}}-\frac{1}{l_{9, n}^{\prime}}+\sqrt{3}\right)=1 .
$$

Proof. Letting $q=e^{-\pi \sqrt{n / 9}}$ in (1.5) and (1.6), we find that $P=\sqrt{3} l_{9, n}^{\prime}$ and $Q=\sqrt{3} l_{9, n}$ in Theorem 3.13. Rewriting (3.13) in terms of $l_{9, n}$ and $l_{9, n}^{\prime}$, we complete the proof.

Theorem 3.15. If $P=\frac{\psi(q)}{q \psi\left(q^{9}\right)}$ and $Q=\frac{\psi\left(q^{2}\right)}{q^{2} \psi\left(q^{18}\right)}$, then

$$
\frac{P}{Q}+\frac{Q}{P}+2=P+\frac{3}{P} \text {. }
$$

Proof. By Lemma 2.2(i) and (iii),

$$
P=\sqrt{\frac{z_{1}}{z_{9}}}\left(\frac{\alpha}{\gamma}\right)^{1 / 8} \text { and } Q=\sqrt{\frac{z_{1}}{z_{9}}}\left(\frac{\alpha}{\gamma}\right)^{1 / 4},
$$

where $\gamma$ has degree 9 over $\alpha$. Thus we have

$$
\frac{P^{2}}{Q}=\sqrt{\frac{z_{1}}{z_{9}}} \text { and } \frac{Q}{P}=\left(\frac{\alpha}{\gamma}\right)^{1 / 8} .
$$

By Lemma 2.4(iv) and (v),

$$
\frac{P}{Q}+\left(\frac{1-\gamma}{1-\alpha}\right)^{1 / 8}-\frac{P}{Q}\left(\frac{1-\gamma}{1-\alpha}\right)^{1 / 8}=\frac{P^{2}}{Q}
$$

and

$$
\frac{Q}{P}+\left(\frac{1-\alpha}{1-\gamma}\right)^{1 / 8}-\frac{Q}{P}\left(\frac{1-\alpha}{1-\gamma}\right)^{1 / 8}=\frac{3 Q}{P^{2}} .
$$

Combining and rewriting above two equations in terms of $P$ and $Q$, we complete the proof.

Using the definition of $l_{k, n}^{\prime}$, we have the following: 
Corollary 3.16. For every positive real number n, we have

$$
\sqrt{3}\left(l_{9, n}^{\prime}+\frac{1}{l_{9, n}^{\prime}}\right)=\frac{l_{9, n}^{\prime}}{l_{9,4 n}^{\prime}}+\frac{l_{9,4 n}^{\prime}}{l_{9, n}^{\prime}}+2 .
$$

Proof. Letting $q=e^{-\pi \sqrt{n / 9}}$ in (1.6), we find that $P=\sqrt{3} l_{9, n}^{\prime}$ and $Q=\sqrt{3} l_{9,4 n}^{\prime}$ in Theorem 3.15. Rewriting (3.15) in terms of $l_{9, n}^{\prime}$ and $l_{9,4 n}^{\prime}$, we complete the proof.

4. Evaluations of specific values of $h_{k, n}, h_{k, n}^{\prime}, l_{k, n}$, and $l_{k, n}^{\prime}$

In this section, we evaluate specific values of $h_{k, n}, h_{k, n}^{\prime}, l_{k, n}$, and $l_{k, n}^{\prime}$ for some positive real numbers $k$ and $n$ by using the explicit relations and formulas established in Section 3.

Theorem 4.1. We have

$$
h_{9,1}^{\prime}=\frac{1}{2}(1-\sqrt{2} \sqrt[4]{3}+\sqrt{3}) .
$$

Proof. Letting $n=1$ in (3.4) and using $h_{9,1}=1$ from Lemma 2.6, we find that

$$
(1-\sqrt{3}) h_{9,1}^{\prime 2}+2 h_{9,1}^{\prime}+1-\sqrt{3}=0 .
$$

Solving for $h_{9,1}^{\prime}$ and using the fact $h_{9,1}^{\prime}<1$, we complete the proof.

Theorem 4.2. We have

(i) $h_{9,4}=5-3 \sqrt{2}+3 \sqrt{3}-2 \sqrt{6}+\frac{\sqrt[4]{3}}{\sqrt{2}}(5-4 \sqrt{2}+3 \sqrt{3}-2 \sqrt{6})$,

(ii) $h_{9,9}=2-\sqrt{3}+\frac{\sqrt[3]{4}(5-3 \sqrt{3})}{(19-11 \sqrt{3})^{1 / 3}}+(2(19-11 \sqrt{3}))^{1 / 3}$.

Proof. For (i), letting $n=4$ in (3.2), we find that

$$
\sqrt{3}\left(\sqrt{h_{9,4} h_{9,1}^{\prime}}+\frac{1}{\sqrt{h_{9,4} h_{9,1}^{\prime}}}\right)=\sqrt{\frac{h_{9,1}^{\prime}}{h_{9,4}}}+\sqrt{\frac{h_{9,4}}{h_{9,1}^{\prime}}}+2 .
$$

Putting the value of $h_{9,1}^{\prime}$ from Theorem 4.1, solving for $h_{9,4}$, and using the fact $h_{9,4}<1$, we complete the proof.

For (ii), letting $n=1$ in (3.6) and using $h_{9,1}=1$ from Lemma 2.6, we find that

$$
3(2-\sqrt{3})\left(h_{9,9}^{2}-\sqrt{3} h_{9,9}+1\right)=h_{9,9}^{3} .
$$

Solving for $h_{9,9}$ and using the fact $h_{9,9}$ has a real value, we complete the proof.

Theorem 4.3. We have

(i) $l_{3,1}^{\prime}=(2+\sqrt{3})^{1 / 4}$,

(ii) $l_{9,1}^{\prime}=\frac{1}{2}(1+\sqrt{2} \sqrt[4]{3}+\sqrt{3})$, 
(iii) $l_{9,4}^{\prime}=1+\sqrt{3}+\frac{1}{2}(\sqrt{2}+\sqrt{6}) \sqrt[4]{3}$,

(iv) $l_{9,9}^{\prime}=\frac{a+\sqrt[4]{3} \sqrt{a\left(a^{2}-\sqrt{3} a+1\right)}}{\sqrt{3} a-1}$,

where

$$
a=2-\sqrt{3}+\frac{\sqrt[3]{4}(5-3 \sqrt{3})}{(19-11 \sqrt{3})^{1 / 3}}+(2(19-11 \sqrt{3}))^{1 / 3} .
$$

Proof. For (i), letting $n=1$ in (3.12) and using $h_{9,1}=1$ from Lemma 2.6, we find that

$$
\left(l_{3,1}^{\prime 4}-4\right)\left(\frac{1}{l_{3,1}^{\prime 4}}-4\right)=1
$$

Solving for $l_{3,1}^{\prime}$ and using the fact $l_{3,1}^{\prime}>1$, we complete the proof.

For (ii), putting $n=1$ in (3.14) and recalling $l_{9,1}=1$ from Lemma 2.7, we find that

$$
(1+\sqrt{3}) l_{9,1}^{\prime 2}-2(2+\sqrt{3}) l_{9,1}^{\prime}+1+\sqrt{3}=0 .
$$

Solving this equation for $l_{9,1}^{\prime}$ and use the fact that $l_{9,1}^{\prime}>1$, we complete the proof.

For (iii), letting $n=1$ in (3.16), we find that

$$
\sqrt{3}\left(l_{9,1}^{\prime}+\frac{1}{l_{9,1}^{\prime}}\right)=\frac{l_{9,1}^{\prime}}{l_{9,4}^{\prime}}+\frac{l_{9,4}^{\prime}}{l_{9,1}^{\prime}}+2 .
$$

Putting the value of $l_{9,1}^{\prime}$ from the previous result of (i), solving for $l_{9,4}^{\prime}$, and noting the fact $l_{9,4}^{\prime}>1$, we complete the proof.

For (iv), Letting $n=9$ in (3.8), we find that

$$
\sqrt{3}\left(l_{9,9}^{\prime}+\frac{1}{l_{9,9}^{\prime}}\right)=\frac{h_{9,9}}{l_{9,9}^{\prime}}+\frac{l_{9,9}^{\prime}}{h_{9,9}}+2 .
$$

Putting the value of $h_{9,9}$ from Theorem 4.2(ii), solving for $l_{9,9}^{\prime}$, and noting the fact that $l_{9,9}^{\prime}>1$, we complete the proof.

See Theorem 3.3(iii) and (vii) in [9] for alternative proofs for Theorem 4.3(i) and (ii).

Theorem 4.4. We have

(i) $l_{9,4}=\frac{1}{2 \sqrt{2}}(3+\sqrt{2}+\sqrt{3}+\sqrt[4]{3}(1+\sqrt{2}+\sqrt{3}))$,

(ii) $l_{9,9}=2+\sqrt{3}+\frac{\sqrt[3]{4}(5+3 \sqrt{3})}{(19+11 \sqrt{3})^{1 / 3}}+(2(19+11 \sqrt{3}))^{1 / 3}$.

Proof. For (i), letting $n=4$ in (3.10), we find that

$$
\sqrt{3} h_{9,4} l_{9,4}+h_{9,4}-l_{9,4}-\sqrt{3}=0 .
$$


Putting the value of $h_{9,4}$ from Theorem 4.2(i) and solving for $l_{9,4}$, we complete the proof.

For (ii), letting $n=9$ in (3.10), we find that

$$
\sqrt{3} h_{9,9} l_{9,9}+h_{9,9}-l_{9,9}-\sqrt{3}=0 .
$$

Putting the value of $h_{9,9}$ from Theorem 4.2(ii) and solving for $l_{9,9}$, we complete the proof.

\section{Some applications}

We now consider some applications of specific values of $h_{k, n}, h_{k, n}^{\prime}, l_{k, n}$, and $l_{k, n}^{\prime}$ for some positive real numbers $k$ and $n$ to evaluations of the cubic continued fractions and the theta function $\psi$. Recall that the cubic continued fraction is defined by

$$
G(q):=\frac{q^{1 / 3}}{1}+\frac{q+q^{2}}{1}+\frac{q^{2}+q^{4}}{1}+\frac{q^{3}+q^{6}}{1}+\cdots=q^{1 / 3} \frac{\chi(-q)}{\chi^{3}\left(-q^{3}\right)}
$$

for $|q|<1$, where $\chi(q):=\left(-q ; q^{2}\right)_{\infty}$.

Theorem 5.1. We have

(i) $G\left(e^{-\pi}\right)=-1-\frac{\sqrt{3}}{2}+\frac{\sqrt{6} \sqrt[4]{3}}{4}(1+\sqrt{3})$,

(ii) $G\left(e^{-2 \pi}\right)=-\frac{1}{4}(1+\sqrt{3}-\sqrt{6} \sqrt[4]{3})$,

(iii) $G\left(e^{-\pi / \sqrt{3}}\right)=\left(\frac{-5+3 \sqrt{3}}{2}\right)^{1 / 3}$,

(iv) $G\left(-e^{-2 \pi}\right)=-\frac{1}{2}(8-6 \sqrt{2}+5 \sqrt{3}-3 \sqrt{6})+\frac{\sqrt[4]{3}}{4}(12-9 \sqrt{2}+8 \sqrt{3}-5 \sqrt{6})$,

(v) $G\left(-e^{-3 \pi}\right)=2-\sqrt{3}+\frac{\sqrt{3}}{\sqrt[3]{4}}\left((5-3 \sqrt{3})(19+11 \sqrt{3})^{1 / 3}-(19-11 \sqrt{3})^{1 / 3}\right)$.

Proof. For (i), letting $n=1$ in Lemma 2.8(i) and putting the value of $l_{9,1}^{\prime}$ from Theorem 4.3(ii), we complete the proof.

For (ii), letting $n=1$ in Lemma 2.8(iii) and putting the value of $h_{9,1}^{\prime}$ from Theorem 4.1, we complete the proof.

For (iii), letting $n=1$ in Lemma 2.8(ii) and putting the value of $l_{3,1}^{\prime}$ from Theorem 4.3(i), we complete the proof.

For (iv), letting $n=4$ in Lemma 2.8 (iv) and putting the value of $l_{9,4}$ from Theorem 4.4(i), we complete the proof.

For $(\mathrm{v})$, letting $n=9$ in either Lemma $2.8(\mathrm{v})$ and putting the value of $l_{9,9}$ from Theorem 4.4(ii) or Lemma 2.8(iv) and putting the value of $h_{9,9}$ from Theorem 4.2(ii), we complete the proof.

See [4] for alternative proofs for Theorem 5.1(i) and (ii). See also [7] and [9] for alternative proofs for Theorem 5.1(iii) and (iv). 
Theorem 5.2. Let a be as in Theorem 4.3(iv). Then we have

$$
G\left(e^{-3 \pi}\right)=\frac{\sqrt{3} a-1}{1+3^{3 / 4} \sqrt{a\left(a^{2}-\sqrt{3} a+1\right)}} .
$$

Proof. Letting $n=9$ in Lemma 2.8(i) and putting the value of $l_{9,9}^{\prime}$ from Theorem 4.3(iv), we complete the proof.

We end this section by evaluating the values of $\psi\left(-e^{-9 \pi}\right)$ and $\psi\left(e^{-9 \pi}\right)$. Note that the numerical values of $\psi\left(-e^{-n \pi}\right)$ and $\psi\left(e^{-n \pi}\right)$ for $n=1,2,3,4,5,6$ were given in [9] by using Lemma 2.9 .

Theorem 5.3. We have

$$
\psi\left(-e^{-9 \pi}\right)=\frac{\pi^{1 / 4} e^{9 \pi / 8}}{6 \cdot 2^{1 / 12} \Gamma(3 / 4)}\left(-2^{1 / 3}+(5+3 \sqrt{3})(-19+11 \sqrt{3})^{2 / 3}\right) .
$$

Proof. Letting $n=9$ in Lemma 2.9 and putting the value of $l_{9,9}$ from Theorem 4.4(ii), we complete the proof.

Theorem 5.4. Let a be as in Theorem 4.3(iv). Then we have

$$
\psi\left(e^{-9 \pi}\right)=\frac{\pi^{1 / 4} e^{9 \pi / 8}(\sqrt{3} a-1)}{2^{5 / 8} \sqrt{3} \Gamma(3 / 4)\left(a+3^{1 / 4} \sqrt{a\left(a^{2}-\sqrt{3} a+1\right)}\right)} .
$$

Proof. Letting $n=9$ in Lemma 2.9 and putting the value of $l_{9,9}^{\prime}$ from Theorem 4.3(iv), we complete the proof.

\section{References}

[1] B. C. Berndt, Number Theory in the Spirit of Ramanujan, American Mathematical Society, 2006.

[2] _ Ramanujan's Notebooks, Part III, Springer-Verlag, New York, 1991.

[3] , Ramanujan's Notebooks, Part IV, Springer-Verlag, New York, 1994.

[4] H. H. Chan, On Ramanujan's cubic continued fraction, Acta Arith. 73 (1995), 343-355.

[5] R. A. Rankin, Modular Forms and Functions, Cambridge University Press, Cambridge, 1977.

[6] B. Schoeneberg, Elliptic Modular Functions, Springer-Verlag, New York, 1974.

[7] J. Yi, The Construction and Applications of Modular Equations, Ph.D. Thesis, University of Illinois at Urbana-Champaign, 2001.

[8] — Theta-function identities and the explicit formulas for theta-function and their applications, J. Math. Anal. Appl. 292 (2004), no. 2, 381-400.

[9] J. Yi, Y. Lee, and D. H. Paek, The explicit formulas and evaluations of Ramanujan's theta-function $\psi$, J. Math. Anal. Appl. 321 (2006), no. 1, 157-181. 
JINHEE YI

Department of Mathematics and Computer Science

Korea Science ACAdemy of KAIST

Busan 614-822, Korea

E-mail address: jhyi100@kaist.ac.kr

Man Gi Cho

Department of Mathematics and Computer Science Korea Science ACAdemy of KAIST

BusAN 614-822, KoreA

E-mail address: chunjaecho@naver.com

JEONG HWAN KIM

Department of Mathematics and Computer Science Korea Science ACAdemy of KAIST

Busan 614-822, Korea

E-mail address: kjhkim0702@naver.com

SEONG Hoi LeE

Department of Mathematics and Computer Science

Korea Science ACAdemy of KAIST

BUSAN 614-822, KorEA

E-mail address: zeusabcd@naver.com

JAe Myung Yu

Department of Mathematics and Computer Science Korea Science ACAdemy of KAIST

BusAn 614-822, KoreA

E-mail address: kfgzx@naver.com

Dae Hyun Paek

Department of Mathematics Education

Busan National University of Education

Busan 611-736, KoreA

E-mail address: paek@bnue.ac.kr 